\title{
Corrigendum: Cathodoluminescence nano-characterization of semiconductors (2011 Semicond. Sci. Technol. 26 064005)
}

\author{
Paul R Edwards and Robert W Martin \\ Department of Physics, SUPA, University of Strathclyde, 107 Rottenrow, Glasgow \\ G4 0NG, UK \\ E-mail: paul.edwards@strath.ac.uk and r.w.martin@strath.ac.uk
}

In our original paper, we estimated the maximum field of view (FOV) that would result when collecting luminescence over a cone half-angle $u$ and coupling this into a spectrograph with a given $f /$ number and a slit width $d$. Due to the use of the low-angle approximation outwith the paraxial regime, the expression given in Equation 2 used the tangent of the angle rather than the correct sine function. The corrected expression is:

$$
\mathrm{FOV}=\frac{d}{2 \cdot(f / \text { number }) \cdot \sin u}
$$

In the high collection $\left(u \rightarrow 90^{\circ}\right)$ limit, this equation describes a FOV which drops to a value of $d /(2 \cdot f /$ number $)$. Thus for the example cited in our paper $(f / 4, d=25 \mu \mathrm{m})$ the minimum FOV will be $\approx 3.1 \mu \mathrm{m}$, as shown in this amended version of Figure 3 :

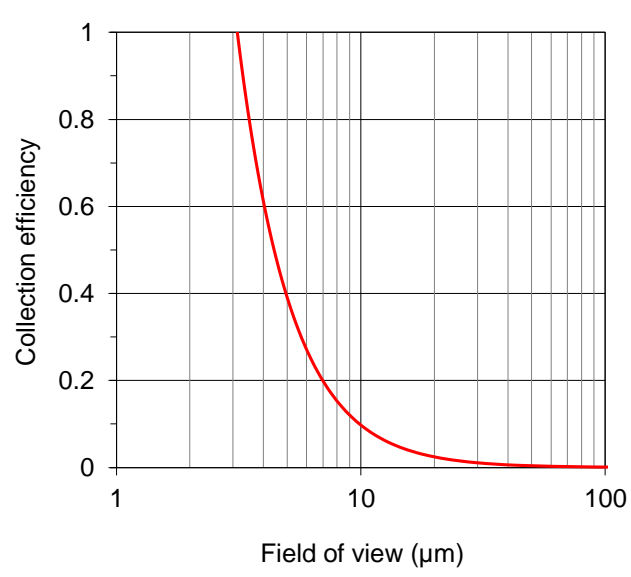

Figure 3. CL collection efficiency versus field of view, calculated for an $f / 4$ spectrometer with $25 \mathrm{\mu m}$ slits.

The conclusion we drew - that a $10 \mu \mathrm{m}$ FOV imposes a collection limit of $\approx 10 \%$ is not altered by this correction. However, the incorrect expression significantly underestimated the FOV possible for higher N.A. collection geometries.

We thank Michael Robertson (Acadia University) and Mathieu Kociak (Université Paris-Sud XI) for bringing this matter to our attention, and for constructive discussions. 